\title{
454 next generation-sequencing outperforms allele-specific PCR, Sanger sequencing, and pyrosequencing for routine KRAS mutation analysis of formalin-fixed, paraffin-embedded samples
}

This article was published in the following Dove Press journal:

OncoTargets and Therapy

3 August 2013

Number of times this article has been viewed

\author{
Annalisa Altimari ${ }^{1}, *$ \\ Dario de Biase ${ }^{2, *}$ \\ Giovanna De Maglio3 \\ Elisa Gruppioni' \\ Elisa Capizzi' \\ Alessio Degiovanni ${ }^{1}$ \\ Antonia D'Errico' \\ Annalisa Pession ${ }^{2}$ \\ Stefano Pizzolitto ${ }^{3}$ \\ Michelangelo Fiorentino ${ }^{1, \#}$ \\ Giovanni Tallini²,\# \\ 'Laboratory of Molecular Oncologic \\ and Transplantation Pathology, \\ S. Orsola-Malpighi Hospital, Bologna, \\ ${ }^{2}$ Laboratory of Molecular Pathology, \\ Anatomic Pathology, Bellaria Hospital, \\ Bologna, ${ }^{3}$ Department of Pathology, \\ S. Maria della Misericordia Hospital, \\ Udine, Italy \\ *These authors contributed equally to \\ this work \\ \#These authors share senior \\ authorship
}

\begin{abstract}
Detection of $K R A S$ mutations in archival pathology samples is critical for therapeutic appropriateness of anti-EGFR monoclonal antibodies in colorectal cancer. We compared the sensitivity, specificity, and accuracy of Sanger sequencing, ARMS-Scorpion (TheraScreen ${ }^{\circledR}$ ) real-time polymerase chain reaction (PCR), pyrosequencing, chip array hybridization, and 454 next-generation sequencing to assess KRAS codon 12 and 13 mutations in 60 nonconsecutive selected cases of colorectal cancer. Twenty of the 60 cases were detected as wild-type KRAS by all methods with $100 \%$ specificity. Among the 40 mutated cases, 13 were discrepant with at least one method. The sensitivity was $85 \%, 90 \%, 93 \%$, and $92 \%$, and the accuracy was $90 \%$, 93\%, 95\%, and 95\% for Sanger sequencing, TheraScreen real-time PCR, pyrosequencing, and chip array hybridization, respectively. The main limitation of Sanger sequencing was its low analytical sensitivity, whereas TheraScreen real-time PCR, pyrosequencing, and chip array hybridization showed higher sensitivity but suffered from the limitations of predesigned assays. Concordance between the methods was $k=0.79$ for Sanger sequencing and $k>0.85$ for the other techniques. Tumor cell enrichment correlated significantly with the abundance of $K R A S$-mutated deoxyribonucleic acid (DNA), evaluated as $\Delta \mathrm{Ct}$ for TheraScreen real-time PCR $(P=0.03)$, percentage of mutation for pyrosequencing $(P=0.001)$, ratio for chip array hybridization $(P=0.003)$, and percentage of mutation for 454 next-generation sequencing $(P=0.004)$. Also, 454 next-generation sequencing showed the best cross correlation for quantification of mutation abundance compared with all the other methods $(P<0.001)$. Our comparison showed the superiority of next-generation sequencing over the other techniques in terms of sensitivity and specificity. Next-generation sequencing will replace Sanger sequencing as the reference technique for diagnostic detection of KRAS mutation in archival tumor tissues.
\end{abstract}

Keywords: colorectal cancer, targeted therapy, KRAS mutations, next-generation sequencing, real-time polymerase chain reaction, pyrosequencing

\section{Introduction}

Monoclonal antibodies against the epidermal growth factor receptor (EGFR) in combination with chemotherapy represent the first example of targeted therapy in colorectal cancer. ${ }^{1}$ The $K R A S$ oncogene is a component of the mitogen-activated protein kinase signal transduction pathway downstream of EGFR. Mutations in codons 12 and 13 of KRAS exon 2 induce stable activation of the GTP-ase KRAS protein, preventing the upstream inhibitory effect of EGFR monoclonal antibodies. ${ }^{2}$
Correspondence: Michelangelo Fiorentino

Istituto Oncologico Addarii, Viale Ercolani 4/2, 40I38, Bologna, Italy

Tel +3905 I636 378।

Fax +3905 16363782

Email michelangelo.fiorentino@aosp.bo.it 
Phase III clinical trials in patients with metastatic colorectal cancer have shown that the response rate to EGFR monoclonal antibodies as well as progression-free and overall survival is poor in those with mutated compared with wild-type exon $2 K R A S .^{3-7}$ Therefore, evaluation of exon 2 $K R A S$ mutations from formalin-fixed, paraffin-embedded pathologic specimens is critical to predict the therapeutic appropriateness of EGFR monoclonal antibodies.

Several techniques are currently available for assessment of $K R A S$ mutations, each with specific advantages and limitations. ${ }^{8}$ Although Sanger sequencing was long considered to be the "gold standard" method for mutational analyses, this method suffers from a low sensitivity threshold and requires at least $30 \%-40 \%$ of neoplastic to non-neoplastic cell ratios (tumor cell enrichment) to detect mutations. A number of alternative technologies based on real-time polymerase chain reaction (PCR, allele-specific, melting curve, PNA clamp) strip assay, chip array, pyrosequencing, and next-generation sequencing have been developed to increase the sensitivity of mutational analyses, allowing investigation of poorly enriched tumor samples below the detection threshold for Sanger sequencing. ${ }^{9-15}$

Pyrosequencing is a sequencing-by-synthesis approach based on sequential addition of dNTPs followed by release of a pyrophosphate molecule that differs from the chain termination dye of the Sanger method. ${ }^{16}$ Pyrosequencing is particularly efficient for investigating mutations in short sequences like KRAS codons 12 and 13. The CE-IVD-marked Anti-EGFR MoAb response ${ }^{\circledR}$ (KRAS status) kit (Diatech Pharmacogenetics, Jesi, Italy) allows tumor cell enrichment as low as $5 \%$, according to the manufacturer. The TheraScreen ${ }^{\circledR}$ kit is a CE-IVD-marked real-time PCR technique based on a combination of allele-specific primers (ARMS) and Scorpion probes suitable for detection of at least $1 \%$ mutant alleles (corresponding to $2 \%$ tumor cell enrichment) in formalin-fixed, paraffin-embedded samples. ${ }^{17}$ The automated CE-marked Infiniti ${ }^{\circledR}$ analyzer (AutoGenomics Inc, Vista, CA, USA) provides a new chip array-based technique designed to detect the most prevalent KRAS codon 12 and 13 mutations with tumor cell enrichment of at least $10 \% .{ }^{18}$ Next-generation sequencing or massive parallel sequencing represents the future of sequencing analyses, and several next-generation sequencing platforms using different sequencing principles are commercially available. The 454 next-generation sequencing platform is based on large-scale parallel sequencing by synthesis similar to pyrosequencing, which is capable of detecting mutated $K R A S$ alleles starting from $1 \%-5 \%$ tumor cell enrichment. Using 454 next-generation sequencing, hundreds of amplicons of the same sequence ("reads") are analyzed in parallel, and the number and percentage of mutated reads can be determined. This provides a quantitative estimation of the relative abundance of the mutated allele. ${ }^{19}$

Several reports have compared the performance of pyrosequencing, real-time PCR, and Sanger sequencing in detecting KRAS mutation for molecular diagnostics. ${ }^{9-11}$ More recently, the Infiniti assay ${ }^{12,13}$ and 454 next-generation sequencing ${ }^{14,15}$ limited to discordant cases have been compared with other techniques.

In the present study, we aimed to compare the sensitivity and accuracy of Sanger sequencing, ARMS/Scorpion realtime PCR, chip array Infiniti, and 454 next-generation sequencing for assessment of KRAS codon 12 and 13 mutations in 60 patients with colorectal cancer. Cases were selected to include uncommon mutations and a wide range of tumor to non-neoplastic cell content.

\section{Materials and methods Sample selection}

From the cases routinely diagnosed with $K R A S$ mutation in the molecular pathology laboratories at the S. Orsola-Malpighi Hospital in Bologna and the S. Maria della Misericordia Hospital in Udine, we selected 60 patients according to the following criteria: histologic confirmation of colorectal cancer; availability of enough formalin-fixed paraffinembedded tumor tissue for the five methods; selection of 40 cases with mutated exon $2 K R A S$ encompassing the largest number of different mutation types, regardless of their known epidemiologic prevalence and 20 tumors with wild-type $K R A S$; and evaluation of mutated samples with tumor cell enrichment ranging from 5\% to $90 \%$.

All cases selected were anonymized, the deoxyribonucleic acid (DNA) re-extracted, and the five sequencing techniques evaluated in a single-blind fashion. Sanger sequencing, real-time PCR, and chip array analysis were performed at S. Orsola-Malpighi Hospital, Bologna, pyrosequencing was carried out at S. Maria della Misericordia Hospital, Udine, and next-generation sequencing at the molecular pathology laboratory at Bellaria Hospital, Bologna. The study was approved by the ethics committee at S. Orsola-Malpighi Hospital.

Sections $10 \mu \mathrm{m}$ thick from representative formalin-fixed, paraffin-embedded blocks of primary $(n=39)$ or metastatic $(n=21)$ colorectal cancer were used. The blocks with the highest proportion of tumor cells over stroma, inflammation, necrosis, and mucinous, normal, or adenomatous colonic tissue were selected by a pathologist (MF) on hematoxylin and eosin-stained slides, circled macroscopically, and 
scalpel-dissected. The number of sections used for DNA extraction varied from one to five, depending on the size of the tumor area. Tumor cell enrichment was expressed as the percentage of neoplastic cell nuclei over the total number of nuclei in the area selected for DNA extraction.

\section{DNA extraction and quantification}

Genomic DNA was extracted using a QIAamp DNA formalin-fixed, paraffin-embedded tissue kit (Qiagen, Hilden, Germany) according to the instructions of the manufacturer with overnight proteinase $\mathrm{K}$ digestion and eluting in $50 \mu \mathrm{L}$ of water. The concentration of the DNA extracted was assessed by real-time PCR using a Quantifiler ${ }^{\circledR}$ kit (Life Technologies, Foster City, CA, USA). All genomic DNA was stored at $-20^{\circ} \mathrm{C}$ until use.

\section{Sanger sequencing}

A 214 bp DNA fragment corresponding to exon 2 of the KRAS gene was amplified by PCR (KRAS-F, 5'-GTGTGACATGTTCTAATATAGTCA-3'; KRAS-R, 5'-GAATGGTCCTGCACCAGTAA-3'; Life Technologies). The annealing temperature was $64^{\circ} \mathrm{C}$. The $\mathrm{PCR}$ products were purified using the MinElute ${ }^{\circledR}$ PCR purification kit (Qiagen) and checked on 2\% agarose gel electrophoresis. Sequencing was performed by applying the same forward and reverse primers and using the BigDye ${ }^{\circledR}$ terminator sequencing kit version 3.0 (Life Technologies) on a 3730XL genetic analyzer (Life Technologies). The sequencing results were interpreted using Chromas software version 1.45 (Technelysium Pty Ltd, Helensvale, Australia).

\section{ARMS/Scorpion real-time PCR}

The TheraScreen KRAS mutation kit (Qiagen, Manchester, UK) was used according to the recommendations of the manufacturer. The assay is designed to detect a wild-type control and the seven most common KRAS exon 2 mutations, ie, Gly12Ala, Gly12Asp, Gly12Arg, Gly12Cys, Gly12Ser, Gly12Val, and Gly13Asp. Real-time PCR was run on a real-time PCR system (7900HT, Life Technologies) using 15-20 ng of genomic DNA for each of the eight mixtures. Data regarding each mutation were interpreted according to the kit manual after curve analysis and calculation of $\Delta \mathrm{Ct}$ values (sample mutation assay $\mathrm{Ct}$ minus sample control assay $\mathrm{Ct}$ ).

\section{Pyrosequencing}

The Anti-EGFR MoAb response (KRAS status) kit was used according to the manufacturer's manual. The test allows identification of all $K R A S$ mutations in codons $12,13,61$, and 146. Briefly, 25-100 ng of genomic DNA were used for analysis of $K R A S$ codons 12 and 13 in PCR volumes of $50 \mu \mathrm{L}$. Real-time PCR was run on a Rotor-Gene ${ }^{\mathrm{TM}} 6000$ (Corbett, Sydney, Australia). Single-stranded DNA templates were immobilized on streptavidin-coated Sepharose highperformance beads (GE Healthcare, Uppsala, Sweden) and then annealed to the sequencing primer using the PyroMark ${ }^{\mathrm{TM}}$ Q96 Vacuum Prep Workstation (Biotage AB, Qiagen). The pyrosequencing reaction was performed on a Pyromark ID instrument (Qiagen) using PyroMark ${ }^{\circledR}$ Pyro Gold Reagents (Qiagen). A negative control and wild-type control were run with each series of samples. Real-time curves and pyrograms were interpreted according to the kit instructions and PyroMark ID software (Qiagen) allowed determination of mutant allelic frequency according to relative peak height.

\section{Chip array hybridization}

Chip array hybridization was performed using the Infiniti KRAS assay on an automated Infiniti analyzer (AutoGenomics Inc) according to the instrument and assay manuals. The assay is designed to detect $K R A S$ mutations in codons 12 and 13, ie, Gly12Ala, Gly12Asp, Gly12Arg, Gly12Cys, Gly12Ser, Gly12Val, Gly12Phe, Gly13Ala, Gly13Cys, Gly13Asp, Gly13Arg, Gly13Ser, and Gly13Val. Briefly, a multiplex amplification was performed using 30-100 ng genomic DNA in a total volume of $20 \mu \mathrm{L}$ on a thermal cycler $(2720$, Life Technologies), followed by an enzymatic cleanup with shrimp alkaline phosphatase and exonuclease I. Incorporation of a fluorescent label by specific primer elongation was then performed within the instrument, followed by hybridization to BioFilm chip microarrays, scanning, and signal reading. One microarray was utilized per each case.

\section{Next-generation sequencing}

Parallel sequencing analysis of $K R A S$ exon 2 was carried out using the 454 GS-Junior ${ }^{\circledR}$ next-generation sequencer platform (Roche Diagnostics, Mannheim, Germany), according to the manufacturer's instructions. Briefly, 10 ng of genomic DNA was amplified using specific $K R A S$ exon 2 primers, and modified with a universal sequencing tail and multiple identifiers nucleotide sequences (Integrated DNA Technologies Inc, Skokie, IL, USA). Negative and nonmutated controls were run per each series of samples. Amplicons were purified and quantified, and an emulsion PCR was performed according to the manufacturer's protocol. A sequencing reaction was performed and the results obtained were analyzed using Amplicon variant analyzer software (Roche Diagnostics, Mannheim, Germany). In all samples analyzed, at least $100 \mathrm{KRAS}$ exon 2 
reads (sequenced amplicons) were obtained, corresponding to an analytical sensitivity of at least $1 \%$.

\section{Statistical analysis}

The kappa coefficient of concordance, and sensitivity, specificity, and accuracy of each test were calculated using 454 next-generation sequencing as the reference method. The Spearman rho correlation was used to correlate the different mutational rate values one to each other and with the percentage of tumor cell enrichment as continuous numerical variables. Data analysis was performed using the Statistical Package for the Social Sciences version 17.0 software (SPSS Inc, Chicago, IL, USA). A two-tailed $P$ value less than 0.05 was used to define statistical significance.

\section{Results \\ Detection of KRAS mutations according to each method}

The 20 wild-type cases were all confirmed using the five different sequencing methods. The number of mutated cases was 34/60 with Sanger sequencing, 36/60 with TheraScreen real-time PCR, 37/60 with pyrosequencing, 37/59 with the chip array assay (in one case, the DNA concentration was too low for Infiniti multiplex amplification, see below), and 40/60 with 454 next-generation sequencing. 454 next-generation sequencing identified the highest number of mutated samples. All mutations identified by 454 next-generation sequencing were confirmed by at least one of the other techniques (Table 1). The 40 mutated cases encompassed the following exon 2 mutation types: Gly12Asp $(n=11)$; Gly12Val $(\mathrm{n}=6)$; Gly12Ala $(\mathrm{n}=4)$; Gly12Cys $(\mathrm{n}=3)$; Gly12Ser ( $\mathrm{n}=2)$; Gly12Arg $(\mathrm{n}=1)$; Gly12Phe $(\mathrm{n}=1)$; Gly13Asp $(n=8)$; Gly13Cys $(n=3)$; and Gly13Val $(n=1)$. Table 1 highlights 13 cases showing discrepancies in the results obtained using the different methods. In particular, six of 40 mutated cases (samples 17, 22, 37, 42, 44, and 45) were not detected by Sanger sequencing because of low tumor cell enrichment $(<30 \%)$. Four of 40 mutated cases not detected by TheraScreen real-time PCR (samples 2, 20, 39 , and 54) fell into specific mutation types not covered by

Table I Summary of cases with discrepant results when Sanger sequencing, real-time PCR with the TheraScreen ${ }^{\circledR}$ kit, pyrosequencing, chip array Infiniti ${ }^{\circledR}$ assay, and 454 next-generation sequencing were used to detect KRAS mutations in formalin-fixed, paraffin-embedded samples

\begin{tabular}{|c|c|c|c|c|c|c|c|}
\hline Sample & $\begin{array}{l}\text { Tumor cell } \\
\text { enrichment }\end{array}$ & $\begin{array}{l}\text { Sanger } \\
\text { sequencing }\end{array}$ & $\begin{array}{l}\text { TheraScreen } \\
\text { real-time PCR }\end{array}$ & Pyrosequencing & $\begin{array}{l}\text { Chip array } \\
\text { assay }\end{array}$ & NGS 454 & Comments \\
\hline 2 & $50 \%$ & Gly|3Cys & WT & Gly|3Cys & GlyI3Cys & GlyI3Cys & $\begin{array}{l}\text { Mutation not in the } \\
\text { TheraScreen kit }\end{array}$ \\
\hline 17 & $20 \%$ & WT & Gly I2Asp & Gly 12Asp & Gly 12Asp & Gly I2Asp & Sanger fails, low enrichment \\
\hline 20 & $90 \%$ & Gly13Cys & WT & Gly I3Cys & Gly13Cys & GlyI3Cys & $\begin{array}{l}\text { Mutation not in the } \\
\text { TheraScreen kit }\end{array}$ \\
\hline 22 & $20 \%$ & WT & Gly I2Val & Gly I2Val & Gly $12 \mathrm{Val}$ & GlyI2Val & Sanger fails, low enrichment \\
\hline 25 & $80 \%$ & Gly12Cys & Gly 12Cys & WT & WT & Gly I2Cys & $\begin{array}{l}\text { Pyrosequencing and chip array } \\
\text { fail, codon II mutation }\end{array}$ \\
\hline 37 & $30 \%$ & WT & Gly|3Asp & Gly13Asp & Gly 13 Asp & Gly|3Asp & Sanger fails, low enrichment \\
\hline 39 & $60 \%$ & GlyI3Val & WT & GlyI3Val & GlyI3Val & GlyI3Val & $\begin{array}{l}\text { Mutation not in the } \\
\text { TheraScreen kit }\end{array}$ \\
\hline 42 & $5 \%$ & WT & Gly I2Val & WT & WT & Gly $12 \mathrm{Val}$ & $\begin{array}{l}\text { Detected only by NGS } \\
\text { and TheraScreen kit, } \\
\text { low enrichment }\end{array}$ \\
\hline 44 & $5 \%$ & WT & Gly|3Asp & Gly|3Asp & Gly13Asp & Gly13Asp & $\begin{array}{l}\text { Only Sanger fails, } \\
\text { low enrichment }\end{array}$ \\
\hline 45 & $5 \%$ & WT & Gly|3Asp & WT & WT & Gly|3Asp & $\begin{array}{l}\text { Detected only by NGS } \\
\text { and TheraScreen kit, } \\
\text { low enrichment }\end{array}$ \\
\hline 52 & $30 \%$ & Gly13Asp & Gly|3Asp & Gly|3Asp & $\mathrm{N} / \mathrm{A}$ & Gly13Asp & $\begin{array}{l}\text { Chip array fails, low DNA } \\
\text { concentration }\end{array}$ \\
\hline 54 & $80 \%$ & Gly|3Cys & WT & Gly|3Cys & Gly|3Cys & Gly13Cys & $\begin{array}{l}\text { Mutation not in the } \\
\text { TheraScreen kit }\end{array}$ \\
\hline 60 & $60 \%$ & Gly|2Phe & Gly I2Val & Gly 12Phe & Gly 12Phe & Gly I2Phe & $\begin{array}{l}\text { Double point mutation } \\
\text { in codon I } 2 \text {, detected by } \\
\text { all methods; TheraScreen kit } \\
\text { fails to detect Phe substitution }\end{array}$ \\
\hline
\end{tabular}


the kit (Gly13Cys and Gly13Val). Pyrosequencing failed to detect mutations in three of 40 cases (samples 42 and 45 ) because of low tumor cell enrichment. In sample 25, the combination of a Gly12Cys plus Ala11Val in codon 11 prevented recognition by pyrosequencing due to impossible primer annealing. The chip array assay failed to identify a mutation in three of 39 cases (sample 52 had low DNA content which was not sufficient for amplification; samples 42 and 45 because of low tumor cell enrichment; and sample 25 for the above-mentioned Gly12Cys plus Ala11Val double mutation). In sample 25, the combination of a Gly12Cys plus Ala11Val in codon 11 prevented recognition by the Infiniti chip array assay because of defective probe hybridization.

\section{Comparison of each KRAS mutation detection method with 454 next-generation sequencing}

Because 454 next-generation sequencing identified the highest number of mutated samples and all mutations called by 454 next-generation sequencing were confirmed by at least one of the other techniques (Table 1), this sequencing method was chosen as the reference for statistical comparison of the other methods. Table 2 describes cross values for the concordance, sensitivity, specificity, and accuracy of each technique. The kappa for concordance of each technique with 454 next-generation sequencing was: Sanger sequencing, $k=0.791$; TheraScreen PCR, $k=0.857$; pyrosequencing kit, $k=0.892$; and chip-array assay $k=0.891$. The sensitivity against 454 next-generation sequencing for the detection of mutations was $85 \%$ (34/40) with Sanger sequencing, 90\% (36/40) with TheraScreen PCR, 93\% (37/40) with pyrosequencing, and 92\% (36/39) with the chip array assay. All four methods, including 454 next-generation sequencing, detected the same 20/20 wild-type cases with $100 \%$ specificity. The proportion of true over total results (accuracy) was 90\%, 93\%, 95\%, and 95\%, respectively, for Sanger sequencing, TheraScreen real-time PCR, pyrosequencing, and chip array assay.

\section{Quantification of mutated DNA}

\section{according to tumor cell enrichment}

We then compared the mean tumor cell enrichment in each histologic sample with the abundance of mutated DNA using the specific quantification criteria provided for each technique, except for Sanger sequencing, which does not allow standardization of the quantity of mutated DNA. In particular, we compared the $\Delta \mathrm{Ct}$ value (calculated as the $\mathrm{Ct}$ of the mutated minus the control assay) for TheraScreen real-time PCR; the percentage of mutation signals for pyrosequencing; the mutated fluorescent signal/total amplicon ratio for chip array assay; and the percentage of mutated alleles for 454 nextgeneration sequencing. As summarized in Table 3, we found a highly significant correlation between the degree of tumor cell enrichment evaluated at histology and the abundance of $K R A S$-mutated DNA for all the techniques, with slightly less significant performance $(P=0.03)$ for real-time PCR. On cross comparison of the different techniques, the best associations were for 454 next-generation sequencing with any of the other three methods, pyrosequencing and chip array $(P<0.001)$, followed by pyrosequencing and TheraScreen PCR $(P=0.001)$ and chip array and TheraScreen PCR $(P=0.004)$.

\section{Discussion}

The choice of methodology with the best cost/effectiveness ratio for detection of somatic tumor mutations, including $K R A S$, is a major challenge for molecular pathology laboratories. Application of advanced techniques to routine molecular diagnostics requires careful evaluation of accuracy and repeatability. The current Italian guidelines for $K R A S$ mutations in colorectal cancer state that a minimum $50 \%$ tumor cell enrichment is required for use of Sanger sequencing, while real-time PCR, pyrosequencing, and strip assay are recommended for less enriched samples. ${ }^{20}$ The European guidelines issued in 2008 recommended no specific test, but stated that "the lower detection limit of mutant signal should be set at $1 \%$ of tumor cells for allelespecific PCR and 25\%-30\% for direct sequencing". ${ }^{21}$ Both guidelines strongly recommend evaluating the repeatability

Table 2 Concordance, sensitivity, specificity, and accuracy of Sanger sequencing, real-time polymerase chain reaction with TheraScreen ${ }^{\circledR}$ kit, pyrosequencing, and chip array Infiniti ${ }^{\circledR}$ assay measured using 454 next-generation sequencing as the reference method

\begin{tabular}{lllll}
\hline Method & Kappa of concordance & Sensitivity & Specificity & Accuracy \\
\hline Sanger sequencing & 0.791 & $34 / 40(85 \%)$ & $100 \%$ & $54 / 60(90 \%)$ \\
TheraScreen $(\Delta \mathrm{Ct})$ & 0.857 & $36 / 40(90 \%)$ & $100 \%$ & $56 / 60(93 \%)$ \\
Pyrosequencing (percent mutated) & 0.892 & $37 / 40(93 \%)$ & $100 \%$ & $57 / 60(95 \%)$ \\
Chip array assay (ratio)* & 0.891 & $36 / 39(92 \%)$ & $100 \%$ & $56 / 59(95 \%)$ \\
\hline
\end{tabular}

Note: *One sample not assessed, values calculated on 59/60 cases. 
Table 3 Statistical correlation between tumor cell enrichment (neoplastic to non-neoplastic cell ratio evaluated on histology sections) and quantification of KRAS mutation abundance according to Sanger sequencing, real-time polymerase chain reaction with TheraScreen ${ }^{\circledR}$ kit, pyrosequencing, chip array Infiniti ${ }^{\circledR}$ assay, and 454 next-generation sequencing

\begin{tabular}{|c|c|c|c|c|c|}
\hline $\begin{array}{l}\text { Method } \\
\text { (quantification criteria) }\end{array}$ & $\begin{array}{l}\text { Tumor cell } \\
\text { enrichment }\end{array}$ & TheraScreen & Pyrosequencing & Chip array assay & NGS 454 \\
\hline Sanger sequencing* & N/A & N/A & $\mathrm{N} / \mathrm{A}$ & $\mathrm{N} / \mathrm{A}$ & $\mathrm{N} / \mathrm{A}$ \\
\hline TheraScreen $(\Delta \mathrm{Ct})$ & 0.030 & - & - & - & - \\
\hline $\begin{array}{l}\text { Pyrosequencing } \\
\text { (\% mutated) }\end{array}$ & 0.007 & 0.002 & - & - & - \\
\hline Chip array assay (ratio) & 0.003 & 0.004 & $<0.001$ & - & - \\
\hline NGS 454 (\% mutated) & 0.004 & $<0.001$ & $<0.001$ & $<0.001$ & - \\
\hline
\end{tabular}

Notes: $P$ value $<0.05$ considered statistically significant. *No precise quantification can be achieved with Sanger sequencing. Abbreviations: N/A, not amplified; NGS, next-generation sequencing.

of each test available in a given molecular laboratory. To our knowledge, this is the first study to compare five different methods for detecting KRAS mutations in colorectal cancer, ie, Sanger sequencing, real-time PCR with the TheraScreen kit, pyrosequencing, chip array Infiniti assay, and 454 next-generation sequencing.

Our data show that sample enrichment is a major limiting factor for Sanger sequencing, while confirming the very high sensitivity of real-time PCR, chip array assay, pyrosequencing, and 454 next-generation sequencing. Had we used a tumor cell enrichment threshold greater than $30 \%$ to analyze our samples, we would have reduced the number of discordances among methods from 13 to seven (Table 1). Equally, our results demonstrate that highly sensitive but mutation-specific approaches, such as the TheraScreen kit, suffer from the use of predesigned assays that do not cover all the possible mutation variants. In our series, five discordances not linked to tumor cell enrichment were related to the inability of the predesigned TheraScreen assay to detect less frequent mutations, such as Gly13Cys and Gly13Val (Table 1). Although pyrosequencing and the Infiniti chip array assay cover a broader spectrum of mutations compared with the TheraScreen kit, they both failed to detect one mutated sample (G12C, sample 25). In this latter case, the simultaneous presence of a codon 11 mutation (revealed by Sanger and 454 next-generation sequencing) prevented detection of a common Gly12Cys mutation due to defective probe hybridization for the Infiniti chip array assay and impossible primer annealing for pyrosequencing. Detection of KRAS double-spot mutations represents a further interpretation problem because it might result in a mistaken wild-type $K R A S$ call when using particular methods. ${ }^{22}$ In our series, the case with the Gly12Phe double point mutation (c.34_35GG > TT; p.G12F sample 60) was considered mutated by all five methods. The TheraScreen kit, which does not include the
Gly12Phe alteration, identified this latter mutation as a Gly12Val (c.35G > T) due to cross reaction of the probe. In this case, the discordance would not have affected therapy, but the occurrence of double mutations further underscores the limitations of any predesigned allele-specific KRAS mutational assay.

Because the results of our study clearly indicate that 454 next-generation sequencing was the single most sensitive and specific method for detection of KRAS mutation, we chose this method to evaluate the relative performance of the other techniques. Comparative evaluation with 454 next-generation sequencing revealed good general concordance for all the methods, and importantly, all methods were $100 \%$ specific (Table 2). Sensitivity and accuracy were acceptable for all methods, but were variably influenced by the different assay designs and the actual number of detectable mutations for each kit. Sanger sequencing was the least sensitive (85\%) and the least accurate (90\%) method, due to the high amount of tumor DNA required to detect the mutation. Sanger sequencing was followed by TheraScreen $(90 \%$ sensitivity and $93 \%$ accuracy) because the kit covers only the seven most frequent mutations in $K R A S$ codons 12 and 13. At equal sensitivity and accuracy (92\%-95\%), pyrosequencing and the chip array assay were unable to detect KRAS mutations in three different cases due to extremely low tumor DNA enrichment in the sample analyzed or to problems related to the predesigned kit.

An original observation of our study is the correlation of tumor DNA enrichment with KRAS mutation abundance, as revealed by the different quantification criteria of each method (Table 3). This correlation was significant for all the techniques, except obviously for Sanger sequencing that cannot provide quantification of mutation abundance. In our hands, pyrosequencing, chip array assay, and 454 nextgeneration sequencing showed the best correlation with tumor cell enrichment, with next-generation sequencing 
also showing the best cross concordance with the other methods (Table 3). The abundance of KRAS mutation is not currently used to guide clinical use of anti-EGFR monoclonal antibodies. However, the abundance of mutated DNA has been recently associated with the clinical response to anti-EGFR tyrosine kinase inhibitors in non-small cell lung cancer. ${ }^{23}$ Introduction of progressively more sensitive methods for detection of KRAS mutations in colorectal cancer raises the issue of the clinical significance of small mutated cell clones over a large background of wild-type tumor burden. If the clinical relevance of quantification of $K R A S$-mutated DNA is demonstrated in the future, it will clearly become necessary to apply methods with the best quantification capability.

On cost-effectiveness grounds, Sanger sequencing is the method with the better defined track record and is the least expensive, relying on instruments and cheap reagents that are widely available. Pyrosequencing, TheraScreen, and chip array assay require expensive reagents and consumables along with purchase or lease of specific instruments required by the different kits. The cost-effectiveness ratio of molecular diagnostic tests depends on a number of factors that can vary considerably, depending on the characteristics of each molecular diagnostic laboratory or the demands of specific institutions. The cost of next-generation sequencing is highly dependent on the number of samples analyzed with each run. Although not in widespread use, we consider that introduction of next-generation sequencing approaches may become the most cost-effective strategy in the near future because of the high throughput capabilities of the instruments that can considerably lower reagent costs.

The aim of this study was to perform a methodologic comparison of a variety of methods currently utilized to diagnose KRAS mutation in colorectal cancer, so we included a number of uncommon mutations, as well as samples with a very wide range of tumor cell enrichment (5\%-90\%). Therefore, our results are influenced by selection criteria that do not necessarily correspond to the everyday practice of most molecular diagnostic laboratories. However, rare KRAS mutations do occur, and should be identified because of their clinical relevance. Also, samples with a large number of non-neoplastic cells and low relative levels of tumor DNA are becoming increasingly common. This is often the case when small biopsies with metastatic deposits or biopsies of tumors that recur after treatment are analyzed. In these instances, lymphocytes, fibrous tissue, and inflammatory cells easily represent the largest proportion of the specimen.
We confirm that tumor cell enrichment is a major factor to be considered when choosing a method to identify KRAS mutations, and show that a molecular diagnostic laboratory cannot rely only on Sanger sequencing to analyze reliably the entire range of formalin-fixed, paraffin-embedded samples currently subjected to KRAS mutational analysis. The analytical sensitivity threshold for Sanger sequencing is quite variable, but generally corresponds to $15 \%-20 \%$ of mutated DNA alleles, ie, $30 \%-40 \%$ of tumor cell enrichment, assuming that the mutation is heterozygous. When dealing with specimens that have tumor cell enrichment lower than $40 \%$ it is mandatory to utilize methods with high analytical sensitivity.

In summary, we demonstrate that all the methods that were compared for detection of KRAS mutation, ie, Sanger sequencing, real-time PCR with the TheraScreen kit, pyrosequencing, Infiniti chip array assay, and 454 next-generation sequencing, have $100 \%$ specificity and overall acceptable sensitivity. We also show that next-generation sequencing performed better than the other methods. It can identify all mutations without being limited by a predesigned set of $K R A S$ mutation targets, has extremely high analytical sensitivity, and can provide a quantitative estimate of the mutational load.

\section{Acknowledgment}

This work was supported by an Italian Government MURST grant (Grant no. 20093XZC57_003) to GT and by grants from the Regione Emilia-Romagna Health Service grant (Progetto Regione-Università, 2010-2012 Area 2), and from the S.Orsola-Malpighi Hospital (Project\# SA43FIOR) to MF and Fondazione Carisbo (Grant no. 2009-0555).

\section{Disclosure}

The authors report no conflicts of interest in this work.

\section{References}

1. Van Cutsem E, Köhne CH, Hitre E, et al. Cetuximab and chemotherapy as initial treatment for metastatic colorectal cancer. $N$ Engl J Med. 2009;360:1408-1417.

2. Banck MS, Grothey A. Biomarkers of resistance to epidermal growth factor receptor monoclonal antibodies in patients with metastatic colorectal cancer. Clin Cancer Res. 2009;15:7492-7501.

3. Van Cutsem E, Lang I, D'Haens G, et al. KRAS status and efficacy in the first-line treatment of patients with metastatic colorectal cancer (mCRC) treated with FOLFIRI with or without cetuximab: the CRYSTAL experience. J Clin Oncol. 2008;26 Supp1 2:Abstr 2.

4. Bokemeyer C, Bondarenko I, Hartmann JT, et al. Efficacy according to biomarker status of cetuximab plus FOLFOX-4 as first-line treatment for metastatic colorectal cancer: the OPUS study. Ann Oncol. 2011;22:1535-1546.

5. Dahabreh IJ, Terasawa T, Castaldi PJ, Trikalinos T. Systematic review: anti-epidermal growth factor receptor treatment effect modification by KRAS mutations in advanced colorectal cancer. Ann Intern Med. 2011;154:37-49. 
6. Van Cutsem E, Köhne CH, Láng I, et al. Cetuximab plus irinotecan, fluorouracil, and leucovorin as first-line treatment for metastatic colorectal cancer: updated analysis of overall survival according to tumor KRAS and BRAF mutation status. J Clin Oncol. 2011;29:2011-2019.

7. Amado RG, Wolf M, Peeters M, et al. Wild-type KRAS is required for panitumumab efficacy in patients with metastatic colorectal cancer. $J$ Clin Oncol. 2008;26:1626-1634.

8. Jimeno A, Messersmith WA, Hirsch FR, Franklin WA, Eckhardt SG. KRAS mutations and sensitivity to epidermal growth factor receptor inhibitors in colorectal cancer: practical application of patient selection. J Clin Oncol. 2009;27:1130-1136.

9. Gao J, Li YY, Sun PN, Shen L. Comparative analysis of dideoxy sequencing, the KRAS strip assay and pyrosequencing for detection of KRAS mutation. World J Gastroenterol. 2010;16:4858-4864.

10. Sundström M, Edlund K, Lindell M, et al. KRAS analysis in colorectal carcinoma: analytical aspects of pyrosequencing and allele-specific PCR in clinical practice. BMC Cancer. 2010;10:660-669.

11. Franklin WA, Haney J, Sugita M, Bemis L, Jimeno A, Messersmith WA. KRAS mutation: comparison of testing methods and tissue sampling techniques in colon cancer. J Mol Diagn. 2010;12:43-50.

12. Bihl MP, Hoeller S, Andreozzi MC, et al. KRAS mutations testing in colorectal cancer: comparison of the results obtained using 3 different methods for the analysis of codons G12 and G13. Diagn Mol Pathol. 2012;21:14-23.

13. French D, Smith A, Powers MP, Wu AH. KRAS mutation detection in colorectal cancer by a commercially available gene chip array compares well with Sanger sequencing. Clin Chim Acta. 2011;412: 1578-1581.

14. Lee S, Brophy VH, Cao J, et al. Analytical performance of a PCR assay for the detection of KRAS mutations (codons 12/13 and 61) in formalin-fixed paraffin-embedded tissue samples of colorectal carcinoma. Virchows Arch. 2012;460:141-149.
15. Gonzalez de Castro D, Angulo B, Gomez B, et al. A comparison of three methods for detecting KRAS mutations in formalin-fixed colorectal cancer specimens. Br J Cancer. 2012;107:345-351.

16. Ronaghi M, Uhlén M, Nyrén P. A sequencing method based on real-time pyrophosphate. Science. 1998;281:363-365.

17. Cross J. DxS Ltd. Pharmacogenomics. 2008;9:463-467.

18. Autogenomics.com [homepage on the Internet]. Autogenomics Inc.; http://www.autogenomics.com/cancer_KRAS-BRAF.php. Available from: http://www.autogenomics.com/. Accessed June 18, 2013.

19. 454.com. 454.com [homepage on the Internet]. 454 Life Sciences, a Roche Company; http://454.com/downloads/GSJuniorSystem_Brochure. pdf. Available from: http://www.454.com/. Accessed June 18, 2013.

20. Aiom.it [homepage on the Internet]. Associazione Italiana di Oncologia Medica; http://www.aiom.it/area+pubblica/area+medica/ prodotti+scientifici/position+paper/Raccomandazioni $\% 20$ per $\% 20$ 1 'analisi $\% 20$ mutazionale $\% 20$ del $\% 20$ gene $\% 20$ KRAS $\% 20$ nel $\% 20$ carcinoma $\% 20$ del\%20colon-retto/1,1006,1,. Available from: http:// www.Aiom.it/. Accessed June 18, 2013.

21. van Krieken JH, Jung A, Kirchner T, et al. KRAS mutation testing for predicting response to anti-EGFR therapy for colorectal carcinoma: proposal for an European quality assurance program. Virchows Arch. 2008;453:417-431.

22. Hostein I, Lamy A, Faur N, et al. The detection of double mutations in KRAS depends on the mutation-detection assay used. Clin Chem. 2011;57: 1077-1079.

23. Zhou Q, Zhang XC, Chen ZH, et al. Relative abundance of EGFR mutations predicts benefit from Gefitinib treatment for advanced non small cell lung cancer. J Clin Oncol. 2011;29:3316-3321.
OncoTargets and Therapy

\section{Publish your work in this journal}

OncoTargets and Therapy is an international, peer-reviewed, open access journal focusing on the pathological basis of all cancers, potential targets for therapy and treatment protocols employed to improve the management of cancer patients. The journal also focuses on the impact of management programs and new therapeutic agents and protocols on

\section{Dovepress}

patient perspectives such as quality of life, adherence and satisfaction The manuscript management system is completely online and includes a very quick and fair peer-review system, which is all easy to use. Visit http://www.dovepress.com/testimonials.php to read real quotes from published authors. 